\title{
Engineering antibody therapies for protective immunity
}

\author{
Nora Lam, BA, ${ }^{a}$ and Donna L. Farber, $\mathrm{PhD}^{\mathrm{b}, \mathrm{c}}$
}

The current coronavirus disease 2019 (COVID-19) pandemic, caused by the novel coronavirus severe acute respiratory syndrome coronavirus 2 (SARS-CoV-2), has placed at the forefront the need for efficacious antiviral therapies that can be rapidly deployed. In cardiovascular diseases, viral infections are a major cause of myocarditis in younger individuals and remain a serious complication following surgery in vulnerable populations such as infants, elderly patients, and in those undergoing solid-organ transplantation. Myocarditis is caused by ubiquitous viruses such as adenovirus, herpes simplex virus, Epstein-Barr virus (EBV), cytomegalovirus (CMV), influenza, and enteroviruses ${ }^{1}$ and is a serious complication in COVID- $19^{2}$; some children with COVID-19 exhibit an inflammatory syndrome similar to Kawasaki disease. ${ }^{3}$ Seasonal viral respiratory tract infections cause morbidity and mortality in heart and lung transplant recipients and can lead to development of pneumonia in immunocompetent patients undergoing cardiothoracic surgery. ${ }^{4}$ Immunosuppressants used to promote graft survival and reduce transplant rejection increase susceptibility to infections, such as respiratory syncytial virus (RSV) and influenza virus, and lead to reactivation of persistent herpes viruses such as EBV and CMV. RSV is a common respiratory infection in infants and young children and poses a substantial complication in infants and children with pulmonary or congenital heart disease..$^{5}$ As vaccines and antivirals are not available for these virus infections, alternate approaches are required for their treatment and prevention.

Preventing infectious disease through vaccines largely occurs via the humoral immune response and the resultant production of high-affinity, pathogen-specific antibodies by $\mathrm{B}$ cells. Antibodies that provide protection from infection, or sterilizing immunity, are referred to as neutralizing antibodies because they directly inhibit productive infection of host cells. Administration of virusspecific monoclonal antibodies has shown therapeutic efficacy in treating RSV infections; palivizumab, an antibody specific for the $\mathrm{F}$ protein of RSV, has been approved by the Food and Drug Administration for

From the Departments of ${ }^{\mathrm{a}}$ Pathology and Cell Biology, ${ }^{\mathrm{b}}$ Surgery, and ${ }^{\mathrm{c}}$ Microbiology and Immunology, Columbia University Irving Medical Center, New York, NY.

Received for publication Feb 29, 2020; revisions received May 19, 2020; accepted for publication May 19, 2020; available ahead of print June 24, 2020.

Address for reprints: Donna L. Farber, PhD, Columbia Center for Translational Immunology, Columbia University Medical Center, New York, NY 10032

(E-mail: df2396@cumc.columbia.edu).

J Thorac Cardiovasc Surg 2021;161:1358-61

$0022-5223 / \$ 36.00$

Copyright (c) 2020 by The American Association for Thoracic Surgery

https://doi.org/10.1016/j.jtcvs.2020.05.107

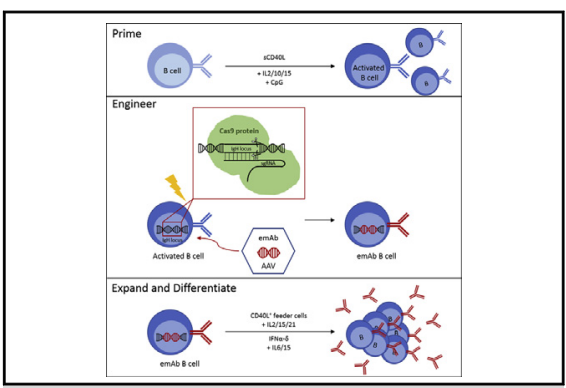

Schematic for expression of engineered monoclonal antibody (emAb) genes into B cells.

CENTRAL MESSAGE

A genetic engineering strategy using CRISPR/Cas9 promotes the expression of virus-specific neutralizing antibodies in B cells, showing great promise for prophylactic therapies for infection.

This Invited Expert Opinion provides a perspective on the following paper: Sci Immunol. 2019;4(35):eaax0644 sciimmunol.aaxo644.

See Commentaries on pages 1362 and 1363 .

prophylaxis of RSV and is given to infants undergoing cardiac surgery at high risk for this infection. ${ }^{6}$ Other monoclonal antibodies specific for HIV, influenza, and CMV have demonstrated protection against infection in vitro and in vivo and are in clinical trials. ${ }^{6}$ While promising, monoclonal antibody treatments require repeated infusions to maintain protection and are costly to produce.

A less-costly and more-durable alternative to provide protective antibodies is viral transduction of B cells with a vector containing genes that encode a protective antibody, enabling long-term persistence of the cells and continuous secretion of neutralizing antibodies into circulation. ${ }^{6}$ However, the amount of antibody produced is fixed despite the severity of infection; therefore, these antibodies are not always protective. Normally, memory B cells express a membrane-bound form of antibody that enables them to rapidly respond and differentiate into additional antibodysecreting cells upon infection, and none of the currently available strategies properly mimic this protective B-cell 
response. Antibodies consist of 2 protein chains (heavy and light chain), undergo class switching to generate different isotypes such as $\operatorname{IgG}, \operatorname{IgA}$, and $\operatorname{IgE}$, and also undergo somatic mutation in the variable region during B-cell development that results in affinity maturation of the antibody response. All of these key aspects of antibody formation and regulation are difficult to mimic simply by expressing a transfected antibody gene into a B cell.

Previous studies have endeavored to recapitulate this complexity by using various B-cell engineering strategies that target antibody-coding regions. Those strategies include replacing the entire heavy chain locus with a heavy chain VDJ of interest, which shows promise only for antibodies that bind antigen without light chain involvement. ${ }^{7}$ Another strategy is inserting a full light chain of interest into the light chain $\mathrm{V}$ region loci and a secreted version of the heavy chain of interest into the heavy chain $\mathrm{V}$ region loci. ${ }^{8}$ None of these strategies, however, inhibit expression of the endogenous antibody.

To address the need for improved antibody therapies that promote immune protection and maintain intrinsic antibody regulation and maturation, Moffett and colleagues ${ }^{9}$ developed a genetic engineering strategy that allowed for the expression of pathogen-specific antibodies in mouse and human B cells. A schematic outlining their strategy is shown in Figure 1. Using highly versatile CRISPR/Cas9 technology, they expressed engineered monoclonal antibody (emAb) genes into B cells while at the same time silencing expression of the endogenous antibody genes. They achieved this by implementing cutting-edge CRISPR/CAs9 gene-editing technology to replace the endogenous antibody genes with the emAb cassette consisting of genes encoding a pathogen-specific antibody. CRISPR/Cas9 ribonucleoprotein and cassette delivery was applied to both mouse and human B cells, and the functional efficacy of the engineered cells was validated in vivo in a mouse infection model.

CRISPR/Cas9 technology is a highly efficient approach for gene editing and derives from an intrinsic defense mechanism of bacteria and archaea, which introduces double-strand breaks in foreign DNA. ${ }^{10}$ Employing this technology in eukaryotic cells requires 2 components: Cas9 and a single-guide RNA (sgRNA) that is complementary to the target sequence of interest. ${ }^{10}$ The Cas9 and sgRNA can be complexed in vitro, forming a ribonucleoprotein, and delivered to cells by electroporation. By designing guide RNAs for regions of interest, it is possible to target and modify expression of any gene in the genome. In the study Moffett by colleagues, the authors generated emAb cassettes encoding a neutralizing antibody specific for RSV, HIV, influenza, or EBV, and efficiency was assessed by measuring the cells' ability to bind viral antigen. The sgRNA targeting the IgH region was highly effective in achieving achieved insertions or deletions (indels) in genomic DNA. ${ }^{9}$ In addition, emAb expression efficiency ranged from $5 \%$ to more than $60 \%$ for different antiviral emAbs (Flu, EBV, HIV). ${ }^{9}$ These results indicate that the CRISPR-targeted antibody engineering was efficient and robust.

Next, the authors used a murine model of infection to assess the ability of these engineered $\mathrm{B}$ cells to respond to infection. After confirming that their RSV emAb B cells bound RSV F antigen, the authors transferred these cells into wild-type mice and were able to detect RSV-specific antibodies in the serum from the mice for several weeks after transfer. Moreover, RSV challenge of mice with the RSV-specific emAb B cells resulted in complete viral clearance, providing compelling evidence that these engineered B cells were therapeutically efficacious. ${ }^{9}$ A final parameter examined in this study was the durability of antibody production by the emAb B cells. For this assessment, the authors transferred emAb B cells into immunodeficient $\mathrm{RAG}^{-/}$-mice, which lack B and T cells, and found that the mice had near-complete protection when challenged intranasally 12 and 87 days after RSV-emAb B cell transfer. ${ }^{9}$ Altogether, the authors demonstrated that their emAb B cells provided near-complete protection against infection in wild-type and immunodeficient mice, demonstrating great promise of this approach.

Overall, this study provides a solid foundation toward the development of an antibody-mediated therapy that mimics the natural protective B-cell response. Their strategy enables insertion of both antibody light chain and heavy chain segments into the intronic region of the $\mathrm{IgH}$ locus, facilitating expression of protective antibodies driven by endogenous regulatory elements. They further demonstrate that their emAb engineering strategy enabled secretion of neutralizing antibodies that provided near-complete protection against infectious challenge in mice. This demonstration of engineered replacement of endogenous antibody gene expression with an engineered antibody is a highly promising and targeted approach for promoting protective immunity, particularly against pathogens for which no vaccines currently exist.

There are some limitations and caveats of this approach that should be considered for translating to humans. One limitation is that the emAb B cells did not increase antibody production in response to infection in mouse hosts, failing to mimic the magnitude and kinetics of a natural protective B-cell response. Serum antibody levels produced by the emAb B cells were stable for a limited period of time, and more studies and optimization are needed for more prolonged persistence beyond months in vivo.

The use of CRISPR/Cas9 technology for generating optimally engineered immune cells is very promising; however, off-target effects can occur, such as gene modifications in DNA regions outside the target gene, due 


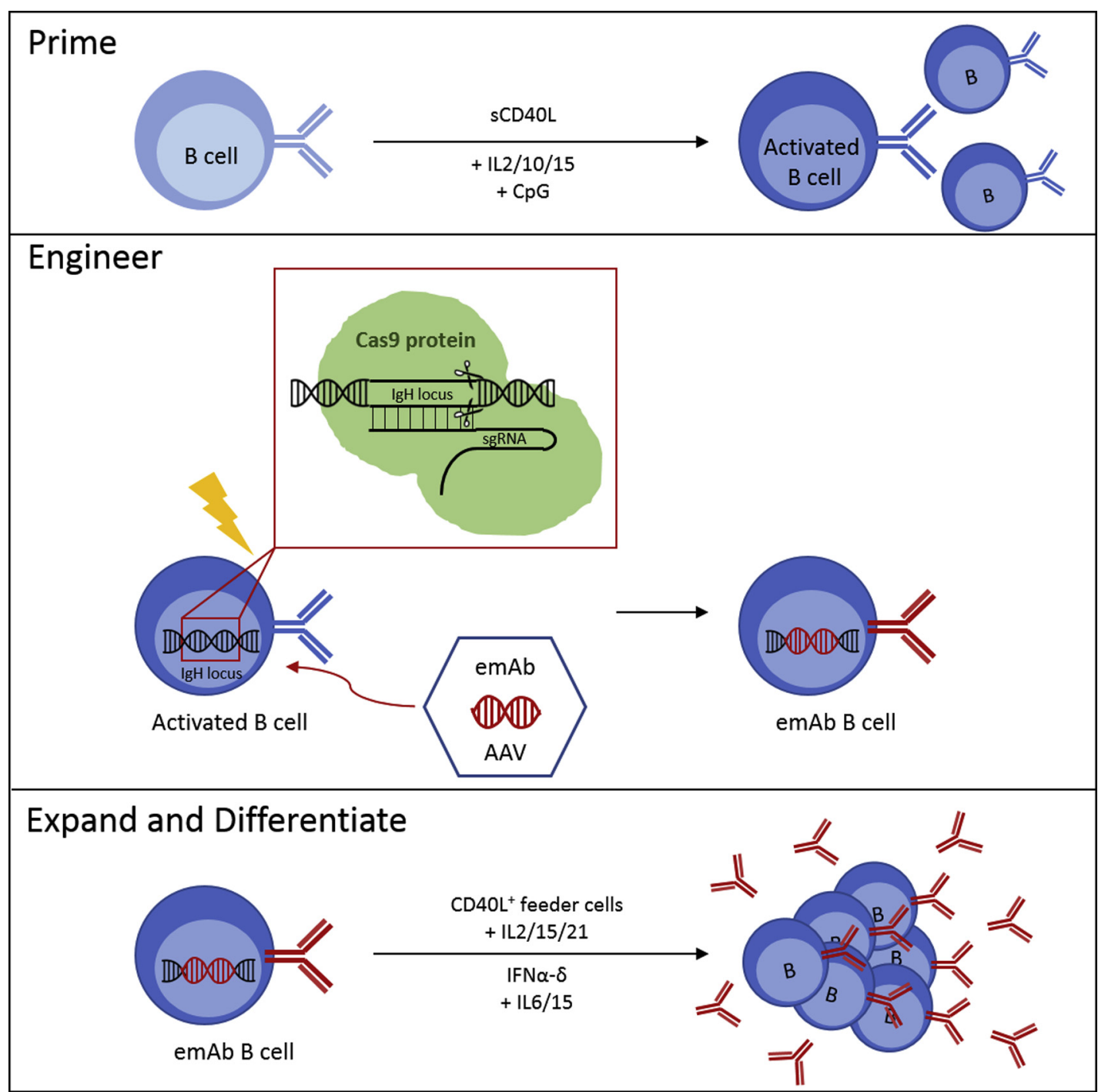

FIGURE 1. Schematic of human B-cell engineering protocol. B cells are primed before engineering with a cocktail of cytokines including IL-2, IL10, and IL-15 (IL2/10/15), multimerized CD40 ligand (sCD40L), and CpG dinucleotides. After 48 hours of culture, the resultant activated B cells are electroporated with CRISPR/Cas 9 complexed with an sgRNA targeting the IgH locus to silence endogenous antibody expression. The emAb of interest is then introduced by incubating the cells with AAV encoding the emAb. Finally, the emAb B cells are then induced to expand and differentiate into antibody-secreting cells through additional culture with feeder cells expressing CD40L and cytokines. $I L$, Interleukin; $s g R N A$, single guide RNA; emAb, engineered monoclonal antibody; $A A V$, adeno-associated virus; $I F N$, interferon.

to sequence similarities within the human genome. Currently, detecting off-target cutting with high sensitivity is challenging to achieve; detection methods have various limitations and biases. ${ }^{11}$ In addition, there is also a significant risk of on-target mutagenesis, such as deletions, insertions, inversions, and translocation events distal and kilobases away from the cut site. ${ }^{12}$ At present, CRISPR/ Cas9-engineered cells must be sequenced comprehensively before they can be transplanted into humans. These caveats may be more theoretical, as a recent phase I safety and efficacy study in patients with cancer found that $\mathrm{T}$ cells genetically modified using CRISPR/Cas9 were well tolerated in human recipients, with mild toxicity and minimal immunogenicity. ${ }^{13}$ Although observations over longer time periods will be necessary, these early results show great promise for the feasibility of using CRISPR/Cas9 gene editing in human therapies. Moreover, given the high potential and broad applicability of CRISPR/Cas9 technologies for therapies, it is anticipated that Cas9 variants with increased specificity and techniques to reduce or eliminate off-target effects are likely to be developed in the near future. 


\section{FUTURE DIRECTIONS}

The approach developed by Moffett and colleagues could be adapted to clinical management and treatment of infections assisted with cardiovascular surgery. The expansion and generation of engineered B cells takes only 2 to 3 weeks and therefore could be incorporated into treatment or prophylaxis with surgical patients. At several weeks before surgery, blood would be obtained, and B cells cultured and expanded ex vivo, for subsequent CRISPR/ Cas9 gene editing. These emAb $\mathrm{B}$ cells could be subsequently administered to patients in the early postoperative period to protect from infections during the most vulnerable stage. Alternately, engineered B cells could be cryogenically preserved, for future use and/or recurrent infections. In this way, a bank of personalized, improved, and fortified immune cells can be stored for future clinical needs.

This approach could be applied in the clinical setting to treat viral diseases where antiviral drug treatments are lacking, such as the viruses that cause viral myocarditis, and for new pandemic viruses, until a vaccine is developed. Convalescent plasma infusions are already being implemented in severe cases of COVID-19, demonstrating the potential significance and efficacy of antibody therapies. ${ }^{14}$ Plasma from recovered donors with COVID-19 contain IgG antibodies specific to the SARS-Cov-2 virus, which can promote viral clearance and neutralization to improve clinical outcomes in recipients. ${ }^{14}$ Using the approach developed by Moffett and colleagues, B cells could be engineered to express and secrete antibodies specific to SARS-CoV-2. A drawback of the scalability of this approach is the 2- to 3-week timeline for patient-specific B-cell engineering. However, if patient-specific B cells could be replaced with universal-donor cells, cells expressing neutralizing antibodies against various viruses can be cryogenically preserved and rapidly administered to any patient. Overall, this approach could be a promising method for effective in vitro preparation of adoptive cellular therapies against pathogens for which no vaccines or antiviral treatments currently exist.

Since more durable persistence of engineered B cells and antibodies occurred in immunocompromised mouse hosts, it is possible that such adoptive immunotherapy may be more successful with patients undergoing immunosuppression or depletional therapies before solid-organ or hematopoietic transplantations. Immunosuppression leading to lymphopenia can create needed "space" for engraftment of adoptively transferred, transfected B cells.
In addition, this therapy could be particularly suited to patients in the early neonatal period when few memory $\mathrm{B}$ cells are present. The presence of engineered B cells could be monitored in patients based on the protein tag engineered into the specific antibodies. Together, generating optimally engineered B cells can improve humoral immunity and represents a personalized medicine, targeted approach to improve the health of individuals, through immune system engineering.

\section{Conflict of Interest Statement}

The authors reported no conflicts of interest.

The Journal policy requires editors and reviewers to disclose conflicts of interest and to decline handling or reviewing manuscripts for which they may have a conflict of interest. The editors and reviewers of this article have no conflicts of interest.

\section{References}

1. Pollack A, Kontorovich AR, Fuster V, Dec GW. Viral myocarditis-diagnosis, treatment options, and current controversies. Nat Rev Cardiol. 2015;12:670-80.

2. Hendren NS, Drazner MH, Bozkurt B, Cooper LT Jr. Description and proposed management of the acute COVID-19 cardiovascular syndrome. Circulation 2020;141:1903-14

3. Cheung EW, Zacharian P, Gorelik M, Boneparth A, Kernie SG, Oange JS, Milner JD. Multisystem inflammatory syndrome related to COVID-19 in previously healthy children and adolescents in New York City. JAMA. June 8, 2020 [Epub ahead of print].

4. Avery RK, Longworth DL. Viral pulmonary infections in thoracic and cardiovascular surgery. Semin Thorac Cardiovasc Surg. 1995;7:88-94.

5. Checchia PA, Paes B, Bont L, Manzoni P, Simões EA, Fauroux B, et al. Defining the risk and associated morbidity and mortality of severe respiratory syncytial virus infection among infants with congenital heart disease. Infect Dis Ther 2017;6:37-56.

6. Salazar G, Zhang N, Fu T-M, An Z. Antibody therapies for the prevention and treatment of viral infections. NPJ Vaccin. 2017;2:19.

7. Voss JE, Gonzalez-Martin A, Andrabi R, Fuller RP, Murrell B, McCoy LE, et al. Reprogramming the antigen specificity of B cells using genome-editing technologies. Elife. 2019;8:e42995.

8. Greiner V, Bou Puerto R, Liu S, Herbel C, Carmona EM, Goldberg MS. CRISPR-Mediated editing of the B cell receptor in primary human B cells. iScience. 2019;12:369-78.

9. Moffett HF, Harms CK, Fitzpatrick KS, Tooley MR, Boonyaratanakornkit J, Taylor JJ. B cells engineered to express pathogen-specific antibodies protect against infection. Sci Immunol. 2019;4:eaax0644.

10. Doudna JA, Charpentier E. Genome editing. The new frontier of genome engineering with CRISPR-Cas9. Science. 2014;346:1258096.

11. Zhang XH, Tee LY, Wang XG, Huang QS, Yang SH. Off-target effects in CRISPR/Cas9-mediated genome engineering. Mol Ther Nucleic Acids. 2015;4: e264.

12. Kosicki M, Tomberg K, Bradley A. Repair of double-strand breaks induced by CRISPR-Cas9 leads to large deletions and complex rearrangements. Nat Biotechnol. 2018;36:765-71.

13. Stadtmauer EA, Fraietta JA, Davis MM, Adam D, Cohen AD, Weber KL, et al CRISPR-engineered $\mathrm{T}$ cells in patients with refractory cancer. Science. 2020;367.

14. Duan K, Liu B, Li C, Zhang H, Yu T, Qu J, et al. Effectiveness of convalescent plasma therapy in severe COVID-19 patients. Proc Natl Acad Sci U S A. 2020; 117:9490-6 